\title{
La Commedia in palcoscenico. Appunti su una ricerca da fare
}

\author{
Marzia Pieri \\ Università di Siena \\ marziapieri@yahoo.it
}

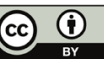

\section{Riassunto}

Da circa due secoli la Divina Commedia è fatta oggetto, in Italia, di letture teatralizzate e di vere e proprie riduzioni drammaturgiche, che hanno raggiunto vaste masse di spettatori, e la cui popolarità non accenna ancora oggi ad esaurirsi. Il fenomeno delle "dantate" - che non conosce equivalenti in altre culture europee - accompagna l'avventura risorgimentale per la conquista dell'indipendenza, ed è legato alla tradizione italiana del grande mattatore romantico: a partire da Gustavo Modena (l'attore mazziniano che intende portare Dante al popolo come veicolo di emancipazione politica), i grandi guitti italiani romantici, naturalistici e poi novecenteschi (di avanguardia o di "tradizione") hanno continuato a recitare il poema, costruendo, per via performativa, una peculiare ecdotica, ancora tutta da studiare. Le celebrazioni dantesche del centenario del 1865 , in una Firenze appena consacrata capitale del Regno d'Italia, segnano un acme di questa fortuna festiva e teatrale.

Parole chiave: Divina Commedia, Risorgimento, Recital, Gustavo Modena, Roberto Benigni, Firenze capitale, cinema muto.

\begin{abstract}
For the last two centuries in Italy, the Divine Comedy has been made subject of dramatized readings and proper dramatic reductions that have reached the bulk of the audience, and whose popularity doesn't yet show any sign of exhaustion. The phenomenon of the 'dantate' - with no equivalent in other European cultures - accompanies the risorgimentale adventure for the sake of gaining independence. This is linked to the Italian tradition of the great romantic 'mattatore': from Gustavo Modena onwards (the Mazzinian actor who attempts to bring Dante to the populace as a vehicle for political emancipation), the great Italian romantic wanderers, first naturalistic and then early twentieth-century (of avant-garde or 'tradition') continued to recite the poem, building, through their performances, a peculiar textual criticism, yet to be studied. The celebrations of Dante's centenary in 1865 , in a Florence that had just been the consecrated capital of the Kingdom of Italy, mark a climax of this festive and theatrical success.
\end{abstract}

Keywords: Divine Comedy, Risorgimento, Recital, Gustavo Modena, Roberto Benigni, Firenze capital, Silent cinema. 
$\mathrm{N}$ ella multiforme storia della fortuna dantesca merita una specifica attenzione il capitolo delle recite del poema, un fenomeno popolare e ricorrente in Italia negli ultimi due secoli, che non accenna ad esaurirsi, e che, anzi, ha attirato l'attenzione (e persino l'emulazione) di critici laureati del calibro di Sermonti, Contini o Raboni ${ }^{\mathrm{I}}$. Il pubblico non sembra mai stancarsene: nell'estate 2014 si tiene a Prato, all'interno dello stadio di rugby, un'Opera music festival dedicato all'Inferno: un kolossal di musica techno, circense e danzato, concepito come sfondo e accompagnamento della declamazione di un vocalist, che poi andrà in tour per l'Italia come prima tappa di un progetto allargato a tutte e tre le cantiche. Il successo nazionale e internazionale delle "improvvisazioni” dantesche di Roberto Benigni continua ad avere tali proporzioni da aver fatto ventilare, nel 2007, l'ipotesi di una sua candidatura al Nobel della letteratura appunto in quanto divulgatore della Commedia (Rainews24, 2I settembre 2007). Ipotesi poi smentita dai fatti, ma non proprio peregrina, perché l'Accademia di Svezia si è sempre mostrata sensibile a cogliere e a promuovere un'idea "larga" di letteratura e di poesia, riconoscendone la presenza e la forza anche all'interno di forme e linguaggi (spesso il teatro) poco tradizionali, e incoronando personaggi scomodi come Dario Fo o Elfriede Jelineck (e prima, magari, Beckett o Pinter), mentre Bob Dylan è in predicato da molti anni per ricevere il premio.

Dall'Ottocento a oggi la Commedia avvince gli spettatori dei teatri, delle arene e della televisione in un paese altrimenti tiepido e distratto verso la poesia e i poeti. In questo senso Dante costituisce una precoce anomalia già a partire dalle letture di Boccaccio in Orsammichele, sollecitate ai Priori da alcuni cittadini fiorentini; la sua leggenda fiorisce nei repertori sentenziosi che gli assegnano i canterini popolari o negli exempla dei predicatori religiosi; personaggi ed episodi del poema, compendiato in terzine già poco dopo la morte dell'autore, migrano più tardi nel teatro, nella narrativa o nell'iconografia, come accade ad Amleto, ad Ariel o a Giulietta e Romeo. Un tipo di popolarità che Dante condivide soltanto con Shakespeare, ma che, nella peculiare tradizione italiana di queste letture drammatizzate del poema - di cui Benigni

I. Negli anni 199I-93 Giovanni Raboni cura per la RAI un ciclo di letture della Commedia; Vittorio Sermonti inizia il 20 ottobre 1992 un'altra lettura radiofonica integrale del poema, frutto di un lungo lavoro svolto con la consulenza di Gianfranco Contini (e sfociato nella pubblicazione dell'edizione Rizzoli curata da entrambi); nel 1995 il critico inizia le sue letture integrali del poema nella basilica di San Francesco a Ravenna, presto replicate con straordinario successo in giro per l'Italia su sfondi suggestivi, come i mercati traianei, o il Pantheon a Roma; il cenacolo di Santa Croce a Firenze; Santa Maria delle Grazie a Milano o Santo Stefano a Bologna. Ricordiamo che il finale dell'ultima edizione 2014 della "Repubblica" delle idee, organizzato a Napoli dal quotidiano romano, è stato animato da una conversazione fra Eugenio Scalfari e Benigni, che si è conclusa con la recita dell'orazione di Ulisse (If. XXVI). Sulla fortuna scenica novecentesca di Dante si rimanda a Giammusso (1994). 
è un diretto epigono -, resta un unicum senza equivalenti fuori d'Italia, dove nessuno si è mai sognato di recitare al popolo Il paradiso perduto, il Faust, o Eugenio Onegin ... ${ }^{2} \mathrm{Ci}$ si può chiedere se un tale slittamento scenico della fruizione sia legato ad un eventuale, specifico carattere concreto e "corporeo" del linguaggio dantesco; se abbia a che fare con un patrimonio identitario nazionale (per quanto, in un paese come l'Italia, sempre fragile e minacciato); se derivi dalla tradizione inaffondabile del grande attore; o magari se sia solo una moda, un ennesimo caso di divulgazione culturale "intelligente", alla fine inspiegabile come i flussi oceanici di visitatori in certe grandi mostre... Di sicuro le proporzioni e la durata del fenomeno sono tali da meritare un'indagine, come un capitolo a sé stante del grande paratesto critico dantesco, al cui interno si potranno individuare di volta in volta - in parallelo alla vicenda storica circostante - coincidenze, continuità o scarti sempre significativi; dopo due secoli di disparate performance, recitare la Commedia è diventato a tutti gli effetti un esercizio ermeneutico che ha costruito legittime, per quanto anomale, interpretazioni: non per via di glosse, scelte redazionali o note a piè di pagina, ma di ritmi espressivi, di pause, di enfasi o di espunzioni, applicate a una testualità di natura fisica, gestuale, iconica e sonora.

Questa vicenda è cominciata in età risorgimentale in chiave politica rivoluzionaria, ma si è consolidata e ufficializzata dopo l'unificazione italiana in tutt'altri termini; nel frattempo Dante è diventato un pezzo fisso nel repertorio e nella formazione dell'attore italiano (maschio di solito, ma non sempre) e un capitolo di routine della manualistica recitativa, con cui tutti i nostri maggiori interpreti, prima o poi, hanno imparato a misurarsi. La storia di questi esercizi di stile compiuti sul poema dagli attori - romantici e naturalisti, e poi novecenteschi, di avanguardia o di tradizione - che lo hanno antologizzato in scena ciascuno a suo modo, è ancora in gran parte da scrivere: da Giovanni Emanuel a Giacinta Pezzana; da Ermete Zacconi a Ruggero Ruggeri; da Vittorio Gassman a Carmelo Bene; da Giorgio Albertazzi a Giancarlo Sbragia; da Arnoldo Foà a Enrico Maria Salerno, a Federico Tiezzi... Benigni è solo l'ultimo arrivato di una lunga serie; il suo marchio di cantastorie popolare e i suoi riferimenti a un presunto background contadino toscano ${ }^{3}$ stanno dentro questa galleria in costume, e andranno forse presi con un certo beneficio d'inventario, un po' come quando Dario Fo si proclamava erede di inesistenti fabulatori popolari del lago Maggiore (Pieri 20Io). Il suo Dante "restituito"

2. Sulla questione si rimanda al celebre saggio di Dionisotti (I97I).

3. Anche se la Pia dei Tolomei è ancora oggi un personaggio-chiave dei maggi e dei bruscelli toscani, la Divina Commedia - diversamente per esempio dai cantari cavallereschi o dalla Gerusalemme liberata - sembra arrivare tardi nelle rappresentazioni folkloriche e spettacolari; ma è anche vero che gli studi degli antropologi e degli studiosi di tradizioni popolari non si sono incrociati a sufficienza con quelli dei letterati e che su questa "assenza" c'è ancora da lavorare; cfr. Battistini (200I) (2008). 
al popolo e carico di allusioni in senso lato "politiche" somiglia in parte a quello di Gustavo Modena, padre nobile del genere e inventore, negli anni ' 30 del diciannovesimo secolo, del format delle "dantate", a cui ci riferiremo in particolare in queste pagine.

Modena era nato a Venezia nel 1803 da una famiglia di attori di fede democratica; il padre Giacomo lo aveva fatto studiare, ma, dopo la laurea in legge e un primo avvio alla professione, il giovane Gustavo aveva a sua volta scelto il palcoscenico, che - insieme alla politica - sarebbe rimasto per sempre la sua grande passione. Ardente repubblicano e patriota, partecipò ai moti antiaustriaci del '2o e del '3i a Padova, a Roma e a Bologna; quando dovette riparare in esilio a Marsiglia, entrò nella Giovane Italia e si legò a Mazzini, che divenne da quel momento il suo mentore e la sua guida politica per tutta un'esistenza segnata da una coerente lotta per l'indipendenza italiana, sempre pagata di persona sul piano privato e professionale, e conclusa in amara solitudine il 20 febbraio del I86I, alla vigilia di un'unificazione monarchica e moderata (proclamata il successivo 17 marzo) per lui, e per quelli come lui, deludente e non condivisibile.

Oltre a imbracciare il fucile tutte le volte che gli sembrava il caso, ad affrontare spesso le privazioni dell'esilio e a schierarsi all'opposizione "di sinistra" nelle assemblee legislative delle effimere repubbliche del I848, Modena si dedicò al teatro con passione e genialità, identificandolo come uno strumento di emancipazione politica delle masse analfabete, fu un attore straordinario e il maestro (ammirato quanto in parte tradito) dei grandi mattatori romantici italiani, come Tommaso Salvini, Ernesto Rossi, Adelaide Ristori o Giacinta Pezzana; tutti suoi allievi, e tutti patrioti che difesero con onore la causa risorgimentale sui palcoscenici d'Europa, ma schierati più comodamente (tranne la garibaldina e indomita Pezzana) dalla parte di Cavour e dei Savoia ${ }^{4}$. Quando incontrò Mazzini, negli anni '3o, Gustavo era già celebre come attore alfieriano e di drammi storici, dove aveva trionfato in particolare nei panni di Paolo nella Francesca da Rimini di Pellico, un best-seller dantesco a fosche tinte che diventò il suo cavallo di battaglia, e che, nel I829, era riuscito a recitare a Padova senza censure grazie all'intercessione della moglie del generale Nugent, pronunciando, nella scena quinta del primo atto, un'appassionata Apostrofe all'Italia acclamata dagli spettatori come una manifesta solidarietà all'autore incarcerato.

Fu il capo della Giovane Italia a orientare e qualificare politicamente il suo slancio eversore e ad ispirargli un progetto culturale di ampio respiro a cui legò in seguito tutto il proprio lavoro: usare il teatro per conquistare alla causa

4. Ricordiamo, ad esempio, che Ernesto Rossi tiene un recital-conferenza a Montevideo il I8 novembre 1879, presentando Dante come precursore dell'Italia concepita da Mazzini e realizzata, congiuntamente e indistintamente, da Cavour, Vittorio Emanuele e Garibaldi (Cfr. Giammusso 1994: II5). 
italiana il "popolo", cioè la piccola borghesia, gli studenti e gli operai delle città, votando l'arte al progresso sociale e sottraendo il palcoscenico alla routine commerciale per farne una palestra di libere opinioni. Dalla mazziniana Filosofia della musica del 1836 Modena derivò il proprio Teatro educatores, la più organica descrizione di un progetto culturale unico nel suo genere all'epoca, successivamente ribadito e perfezionato in una folta serie di opuscoli polemici e satirici. Era un sogno audace e impossibile ("il mio [teatro] è negli spazi oltre la luna"), legato alla profonda consapevolezza che "l'ideale dell'arte non si può conseguire in Italia: non autori, non pubblico, non corona di buoni attori: le tragedie o proibite o ristrette alle quattro più sciape e rifritte eternamente" 7 . Il suo Dante è una sorta di antidoto rispetto a tanto disincanto, sta dentro un'utopia e lo accompagna lungo una frequentazione scenica che si articola nel tempo in varie tappe: dall'idea di recitarlo, alla tentazione conseguente di identificarvisi in quanto esule fuggiasco, all'approdo conclusivo - paradossale ma non troppo - di "riscriverlo" attualizzato in forma di Commedione satirico sul moderno inferno italiano del regime sabaudo.

Ma andiamo con ordine. Nei circoli democratici francesi, inglesi e svizzeri degli anni '3o Modena si trovò a disposizione un terreno fertile e in qualche modo già dissodato: dopo la rivoluzione francese il culto di Dante, accanto a quello di Omero e di Shakespeare, aveva fatto molta strada, per ragioni più ideologiche che filologiche, e il poeta dal linguaggio veemente e appassionato aveva riguadagnato terreno sui suoi tradizionali concorrenti Petrarca e Boccaccio. Alfieri, Monti, Leopardi e il giovane Manzoni avevano cominciato a riconoscerlo come un padre dell'Italia da costruire; Morrocchesi, l'attore alfieriano, aveva scritto nel 1822 il dramma Dante in Ravenna, puntando l'attenzione sulla vittima dell'ingratitudine fiorentina; ma era stato Foscolo a coglierne, dalla prospettiva inglese dell'esilio, tutto il potenziale polemicamente patriottico ${ }^{8}$, mettendo da parte le anguste, eterne questioni della lingua e dello stile su cui in Italia finiva inesorabilmente per arenarsi qualsiasi discussione in materia (Dionisotti 1971: 278). I suoi saggi avevano inaugurato il grande filone del dantismo inglese, alimentato, nel I826, da un altro italiano fuoriuscito reduce dai moti del '2o e del '2I, come Gabriele Rossetti, che a sua volta raccolse l'invito mazziniano a studiare Dante per farlo rivivere, sottraendolo alle glosse disseccate dei grammatici che ne avevano fatto un cadavere?.

5. Il saggio uscì il 3I ottobre I836 sullo stesso giornale, "L'Italiano", che già aveva ospitato le riflessioni di Mazzini sulla riforma necessaria delle scene musicali, riprendendone molti spunti applicati alla scena drammatica (cfr. Mango 20I2: 86).

6. In questi termini si concludevano le pagine del Teatro educatore, (cfr. Meldolesi 1971: 63).

7. Così Gustavo scrive da Bruxelles al padre il I3 ottobre I838 (Grandi I955a: 27).

8. Il suo Discorso sul testo del poema di Dante è del I825, e lo stesso Mazzini ne curerà una riedizione nel 1842.

9. 'O Italiani, studiate Dante. Studiate Dante non su' commenti, non sulle glosse; ma nella 
Prima di lui altri illustri esuli democratici, quali Filippo Mazzei o Lorenzo Da Ponte ${ }^{\mathrm{IO}}$, avevano preso a divulgarne in Inghilterra e negli Stati Uniti quel ritratto idealizzato e libertario che la cultura romantica ottocentesca avrebbe fatto proprio, replicandolo disseminato in pagina, in tela o in marmo in giro per la penisola ${ }^{\mathrm{II}}$.

Quando, dunque, il giovane Modena, a corto di denaro, si inventa dei recital danteschi da salotto con accompagnamento di arpe e violini, con la speranza di racimolare i soldi per un biglietto di ritorno in Italia ${ }^{12}$, non fa che intercettare abilmente un tema familiare al pubblico di élite a cui in quel momento si rivolge, unendo l'utile al dilettevole: guadagnare consensi alla causa italiana e fare cassetta con una produzione agile e a basso costo, ma culturalmente referenziata. Comincia a provarcisi sperimentalmente in Francia e in Svizzera in circuiti privati, dove il sottofondo musicale e l' intensa espressività mimica dell'interprete (per esempio nell'impersonare il conte Ugolino) suppliscono alla difficoltà linguistica degli astanti, proponendo una sorta di pantomima animata di grande impatto emotivo, ma il debutto ufficiale è a Londra, il I7 maggio del I839, all'Her Majesty's Theatre: la locandina propone, in diurna, un Morning Concert e una Italian Declamation (Grandi 1968). In sala, mescolato ad un pubblico aristocratico, c'è anche il principe Napoleone: all'epoca Modena ha 36 anni e si presenta in scena in costume di Dante Alighieri (ormai fissato da una riconoscibile iconografia) mentre detta ad un amanuense le strofe del poema: fra pause, silenzi, tumulti indignati di un cuore appassionato, l'attore si muove fra il boccascena e il fondo palco, costruendo una dinamica scenica che ricostruisce in forma drammatizzata lo stesso processo creativo del poema ${ }^{13}$.

L'idea riscosse un successo straordinario, e da quel momento l'attore continuò, fino a alla fine dei suoi giorni, a replicarla e ad affinarla con poche varianti, talvolta sostituendo la spalla dell'amanuense con una figura fem-

storia del secolo in ch'egli visse, nella sua vita, nelle sue opere. Ma badate: v'ha più che il verso nel suo poema; e per questo non vi fidate a' grammatici e agli interpreti; essi sono come la gente che dissecca i cadaveri; voi vedete le ossa, i muscoli, le vene che formavano il corpo; ma dov'è la scintilla che l'animò?" (Mazzini s.d.).

Io. Si rimanda a Costa (2002).

II. Si veda in proposito il catalogo della mostra (Firenze dicembre 1973/febbraio 1974) Romanticismo storico Celebrazioni per il Centenario di Francesco Domenico Guerrazzi, (Barocchi et al. 1974: 4I-55) ("I grandi personaggi della poesia romantica") e Querci (a cura di (20II)).

I2. Nel I838 un'amnistia gli offre la possibilità di rientrare nel Lombardo-Veneto, dove Gustavo, che si è sempre rifiutato di rivolgere una supplica all'arciduca d'Austria, conta di reinserirsi come direttore di un teatro milanese di cui si progetta l'apertura; a corto di risorse, si sposta da Bruxelles a Londra, dove Mazzini lo ospita e lo aiuta a organizzare lo spettacolo su Dante e Manzoni. Dopo molte traversie rientrerà a Livorno il 4 luglio I839.

13. Quella del poeta al lavoro, che smania e sogna, è quasi un topos recitativo, utilizzato in questi anni a man bassa, per esempio a proposito di Torquato Tasso, un altro scrittore / personaggio assai frequentato sulle scene da Goethe a Goldoni; cfr. Pieri (I995). 
minile che impersonava la Poesia o Beatrice, o in altri casi lasciando il poeta - laico, ghibellino, esule - solo a comporre e leggere i suoi versi davanti a un leggio o nella solitudine rupestre di un paesaggio desolato. L'invenzione non nasce insomma come semplice esercizio di dizione declamatoria, ma come un progetto di spettacolo a tutto tondo, in cui certamente l'istrione è centrale, ma collocato e supportato entro un contesto multimediale (scenografico, musicale, illuminotecnico) via via perfezionato, e si configura ben presto come un vero e proprio nuovo genere drammaturgico, presto imitato da altre compagnie $^{\mathrm{I} 4}$. Le numerose testimonianze che ci restano a proposito delle dantate modeniane sottolineano sempre con ammirazione l'efficacia comunicativa di un tale dispositivo "minimalista", che consente agli spettatori intense integrazioni emotive e fantastiche, come testimonia un recensore del I840:

Egli è Dante che qua e là rivede alcuni passi della Divina Commedia, e gli declama, o piuttosto gli recita seguendo l'impeto della fantasia non già l'ordine del Poema. Il suo abito è in costume, e precisamente conforme, se la memoria non mi tradisce, al quadro che ci rappresenta l'Alighieri nel Duomo di Firenze. Con varj rotoli di carta tra mano egli siede sopra un sasso in luogo romito, selvaggio, che alla tua immaginazione può ben ricordare o gli orrori di Fonte Avellana, o le rovine di Tolmino, o qualsiasi altra solitudine in cui Dante abbia meditato il poema sacro [...] poco lungi da esso una donna, in cui vuolsi forse rappresentare la immortalità, sta incidendo sul bronzo i versi del divino Poeta ${ }^{15}$.

L'efficacia di questi allestimenti, costruiti sul registro del patriottismo e dell'indignazione, era moltiplicata dall' ambigua sovrapposizione, che presto si creò agli occhi degli spettatori, fra attore e personaggio. L'esule Modena cantava l'esule Dante; la loro sofferenza era comune; comune il loro destino di ribelli solitari in una società corrotta; il passato e il presente si intrecciavano, e spesso la recita si concludeva con un'audace ma plausibile "coda" manzoniana: i cori del Carmagnola o dell'Adelchi, o certi brani dei Promessi Sposi (che già Modena aveva ridotto in 5 atti nel 1829 come un Ronconi avant-lettre ${ }^{16}$ ) si sposavano perfettamente con una tale lettura della Commedia; Manzoni, del resto, era altrettanto famoso e politicamente utilizzabile, nonostante le diffidenze dell'attore verso il suo cattolicesimo.

Al suo progetto militante di alta divulgazione, e di pedagogia politica per via teatrale mancavano infatti, fatalmente, testi e personaggi adeguati, che egli tentava di racimolare come poteva, soprattutto ricorrendo ad autori stranieri.

I4. Un'analisi dettagliata e molti documenti di prima mano sull'intera questione fornisce la bella monografia di Petrini (2012: 165-200). E si veda anche il saggio di Puppa (2012).

15. [Sia], Esperimento di declamazione dato da Gustavo Modena, in "Il Ricoglitore di notizie teatrali", I3 giugno I840 (Cfr. Petrini 20I2: I83).

I6. Su questa riduzione drammaturgica del romanzo (che prevede, tra l'altro, l'espunzione del personaggio di fra Cristoforo), cfr. Petrini (2012: 176-I82). 
Modena legge la Commedia come un dramma storico in nuce carico di elementi tragicomici, tramata di dialoghi e di parti narrative ${ }^{17}$; un serbatoio di storie potenzialmente "popolari" tutte legate a un protagonista d'eccezione: Dante è per lui soprattutto il personaggio di cui è eternamente alla ricerca, è l' "eretico mascherato da cattolico"I8 che incarna a pieno titolo la drammaturgia moderna e per tutti, di cui l'Italia avrebbe tanto bisogno, e alla cui mancanza egli tenta, da parte sua, di supplire con una recitazione in grado di riempire di senso e di contenuti teatrali i testi più disparati che gli appaiono idonei alla bisogna. Come fece in campo musicale Verdi - a cui è stato spesso paragonato - Modena si adoprò per cercare di colmare il profondo divario fra la cultura ufficiale e il popolo (Meldolesi 1971: 60), costruendo un immaginario collettivo nuovo, con al centro eroi umanamente accessibili e riconoscibili. Oltre dunque a lavorare in prima persona, traducendo e riadattando per la scena materiali di ogni genere (come del resto facevano da secoli i suoi colleghi attori, sempre in rotta di collisione con i letterati, disinteressati o supponenti nei confronti del palcoscenico), egli sollecita continuamente gli autori amici a fornirgli drammi di questo tipo, per cui, ad esempio, a Giuseppe Vollo, che ne stava componendo uno su fra Dolcino, suggerisce di farlo incontrare e discutere proprio con Dante: "potresti [...] far cadere Dante viaggiatore, reduce da Parigi, nelle mani dei dolciniani, che non lo ammazzano perché gli trovano in saccoccia i versi contro la chiesa di Roma, e Dolcino svillaneggia il poeta perché con logica stolta vuol toglier l'Italia ai Guelfi per regalarla all'Imperatore e mantenere il papato come cosa divina. Dolcino predica che i poeti saranno apostoli di servitù, ruffiani di re"I9.

Il suo Dante, più che un benevolo padre della patria, è un laico, laicissimo censore della politica risorgimentale in corso, come egli non manca di ribadire in molte circostanze, per esempio appaiandolo al Maometto di Voltaire (che aveva tradotto in endecasillabi) in una recita torinese del 1858 , che invita il pubblico ad andare a vedere a teatro per cogliere "nella Mecca araba simboleggiata la gran Puttana dell'Apocalisse e di Dante” (Puppa 20I2: 187). Dagli anni ' 30 fino a pochi mesi dalla morte, Modena non interrompe mai quest' intensa frequentazione del poema, che continua a recitare in chiaroscuro con quanto sta accadendo nella politica risorgimentale. I suoi cavalli di battaglia allineano senza soluzione di continuità la Divina Commedia, il Saul e il Luigi $X I^{2 \circ}$, cucendosi addosso Dante, Alfieri e Delavigne nel segno del tragicomico e dell'invettiva politica.

I7. Si rimanda alla fine analisi di Pietrini (2012).

I8. Cfr. Petrini (2012: 172) sul carattere eretico di Dante secondo Modena.

19. Meldolesi (197I: 76): Il progetto è contenuto in una lettera del giugno I850 (Grandi 1955a: I24-I25).

20. Si veda Randi (20I2). 
Così, con il trascorrere del tempo e dopo la delusione quarantottesca, l'attore aggiorna e corregge progressivamente - rispetto al registro romantico dell'esordio londinese - sia il rapporto con gli spettatori che la scaletta dei brani recitati, selezionandoli di volta in volta in ragione della loro potenziale icasticità scenica e del tasso attualizzante di polemica o di satira che erano in grado di veicolare. Al pubblico dell'Her Majesty's Theatre aveva proposto gli episodi celebri di Paolo e Francesca, del conte Ugolino, di Capaneo, e di Farinata degli Uberti, di Manfredi, dei simoniaci e dei ladri, e ci aveva aggiunto l'invocazione all'Italia e la maledizione dei diavoli, cioè un menù a forti tinte, carico di passioni e di invettive. Più tardi integrerà $o$ alternerà questi pezzi con la Beatrice del XXX canto del Purgatorio, o con l'apostrofe di San Pietro contro la Chiesa corrotta, sempre prediligendo le descrizioni dei supplizi di ladri e falsari o seminatori di discordie atrocemente puniti, come Mosca de' Lamberti, Curione o Bertrand del Bormio ${ }^{21}$. Un pezzo forte, assai ammirato dai recensori, è la tirata contro il potere temporale della Chiesa derivato dalla sciagurata donazione di Costantino del canto XIX dell'Inferno, a proposito della quale in molti testimoniano l'utilizzo di un espediente scenico di grande effetto, significativo del peculiare "trattamento" dantesco operato dall'attore: al verso "Ahi, Costantin, di quanto mal fu matre, / non la tua conversion, $m a$ quella dote", egli simulava una titubanza, e provava la versione corretta " $e$ la tua conversion, $e$ la tua dote" con un'enfasi rabbiosa che strappava gli applausi $^{22}$. La comunicazione profonda del senso era affidata a minimi dettagli scenici molto concreti, come attesta un altro esempio celebre relativo al canto $\mathrm{V}$ dell'Inferno:

al verso "Caddi come corpo morto cade" egli prima di dire "caddi" si arrestava come se cercasse la parola, e nel breve agitarsi della mente per formularlo, la pergamena che teneva in mano gli scivolava inavvertitamente, ma il fruscio comunque lieve di quella caduta pareva che dovesse essere per lui la scintilla che doveva illuminargli la mente, poiché fissando quella cartapecora l'additava dicendo: "Come corpo morto cade"z3.

Per suo tramite il testo diventa vivo: le integrazioni pantomimiche, i chiaroscuri espressionisti venati di macabro, ispirati a una truce ironia e aspramente polemici, le sottolineature di particolari passaggi, le "correzioni" suggerite con disinvoltura in forma di ipotetiche varianti, erano indirizzati al suo autenti-

2I. La struttura delle recite era aggiornata di volta in volta in base alle circostanze; Luigi Rasi riporta, ad esempio, il programma di sala di una serata al teatro del Giglio di Lucca del 7 giugno I840, che offriva: "Francesca da Rimini - Cerbero (canti V-VI) - Ladri tramutati in serpi (canto XXV)- Curio - il Mosca - Bertram del Bormio (XXVIII) - Falsatori - Maestro Adamo (XXIX e XXX) - Lucifero - Bocca - Ugolino (XXXII-XXXIII- XXXIV)" (Rasi 1905: 138).

22. La sequenza è analizzata da Petrini (2012: 187).

23. Andrei (1899: 103). 
co pubblico italiano, molto lontano e diverso dai colti spettatori inglesi del I839: un pubblico non scolarizzato, ma a suo parere perfettamente in grado di recepire la poesia della Commedia, opportunamente mediata dal commento scenico, per farne di diritto un patrimonio proprio, per riprendersela e nutrirsene. Modena non è un attore che voglia abbagliare e commuovere, ma un militante e un pedagogo della scena, che intende far crescere e maturare chi va a teatro, per responsabilizzarlo a pensare in libertà ${ }^{24}$. Il suo stile non punta al sublime estetizzante, ma al grottesco epico, dove i contrasti vivaci e continui sono finalizzati, didatticamente, a uno spiazzamento del destinatario, che lo obblighi a riflettere e criticare; per questo eccelle specialmente nelle parti narrative del poema, di cui offre commenti molto "tecnici" affidati ad "azioni spiegative" (Petrini 2012: 192) in grado di visualizzare le immagini dantesche; egli gioca sull'alternanza, e avvicenda bruscamente, senza soluzione di continuità, gli slanci di spiritualità eroica ai toni acri della satira, tenendosi lontano dalle armonie classicistiche, senza suggerire immedesimazioni sentimentali. La poesia dantesca, del resto, gli offre da questo punto di vista un corrispettivo stilistico ideale: concreto e iconico, il suo linguaggio è perfettamente contiguo al peculiare realismo sterniano dell'attore, remoto dal "naturale", che mescola sapientemente declamazione e recitazione entrando e uscendo in continuazione dal personaggio che interpreta.

Una poetica modernissima quella di Modena - nutrita di etica e non di estetica, e decisamente "contenutista" - sulla cui efficacia presso i destinatari egli non cessa mai di interrogarsi, con lo scrupolo di non sovraccaricare l'enfasi espressionistica, pur necessaria per farsi intendere appieno da tutti: "se sapessi quante volte mi arrabbio con me, per questa recitazione di Dante che mi fa scivolare nel brutto vizio di telegrafare e gesticolare come un lazzarone. Ho sempre paura che il colto pubblico non intenda, e perciò cado in iscimiate"25. Egli non era affatto paternalista o compiacente verso i suoi spettatori, e aveva le idee ben chiare circa la qualità culturale del proprio lavoro. Il suo Dante scenico esordisce romanticamente come poeta di sentimenti privati appassionati e coinvolgenti, ma tende a farsi negli anni sempre più tendenzioso ed epicizzato: "la rappresentazione della Divina Commedia divenne lo spettacolo per eccellenza del culto degli ideali e del rispetto di sé"26, raggiungendo effettivamente grandi masse di pubblico nelle tournées degli anni '40 e 'so, che gli guadagnano fama e ammirazione anche presso gli addetti ai lavori della

24. Nel suo fondamentale studio sociologico sul pubblico teatrale italiano, Bruno Sanguanini dedica un importante paragrafo a "Umanità e ideali sociali in Schiller, Mazzini, Modena" (Sanguanini I989: I04-II3).

25. Cfr. Meldolesi (197I: 95). La citazione è tratta dal testo modeniano Il cattolicismo di Dante, in Grandi (a cura di) (1955b: 303)

26. Un tale passaggio interpretativo non convinse tutti; sulla discussione che si accese in materia fra i contemporanei, cfr. C. Meldolesi (I971: 96). 
letteratura, in genere così poco interessati al teatro. Fino alla fine, come si è detto, continua a recitarla, anche in polemica amara contro i cedimenti mazziniani dei tardi anni '5o (fra il I859 e il I86I i due saranno politicamente distanti), che, anteponendo l'obiettivo dell'unità a quello della repubblica, avevano tragicamente compromesso, a suo giudizio, la causa della libertà. La sua strategia militante e il suo giudizio severo sulla politica italiana restano sempre nitidi e coerenti e quando, nel I860, gli viene offerta una cattedra di declamazione a Firenze (dove già era stato condannato a vent'anni di galera per la sua partecipazione ai fatti del ' $48^{27}$ ), la rifiuta senza esitare, nonostante le ristrettezze economiche in cui versa. Questa parte della vicenda si conclude nell'ultimo decennio della sua vita con un'intensa attività libellistica di marca illuministica e antisabauda, affidata, fra l'altro, al già ricordato Commedione, un bizzarro pastiche a metà strada fra dialogo satirico e commedia, che avrebbe dovuto incorniciare organicamente il suo pensiero; come scrive in una lettera a G. Savon del I856: "i popoli conoscono i loro Guelfi e Ghibellini e gli ipocriti e gli ambiziosi e gli avari e - mutando i nomi sull'Inferno di Dante calcherebbero un Inferno moderno più largo con chieriche, e con re di Francia e di Napoli ecc..., e un Purgatorio veramente purgante ${ }^{28}$ ".

Modena non riuscì a realizzare questa sintesi ambiziosa, ma il progetto - di cui sopravvivono una serie di "pezzi" e molte tracce programmatiche resta assai significativo (Meldolesi I97I: IOI-IIO). Al suo dantismo, peraltro, destinatari qualificati riconobbero, come si è detto, dignità di legittimo esercizio ermeneutico: l'ambizione di mostrare in scena il farsi stesso della poesia affascina i più e anche scandalizza qualcuno; e se c'è chi gli richiede addirittura un di più di verosimiglianza (per esempio invitandolo insistentemente a tagliarsi la barba che avrebbe ostacolato l'identificazione totale con il poeta), altri sono invece capaci di cogliere il nucleo simbolico e allegorico dell'interpretazione, oltre la lettera del quadro scenico:

io non vedeva in lui l'artista, che avesse la pretensione cogli spostamenti delle braccia, colle stonature della voce e cogli sgangheramenti della faccia, di farci la geroglifica rappresentazione degli eventi fantastici della Divina Commedia, ma vedevo nell'artista il poeta che si compiaceva di contemplare la propria creazione, e che, nel ripetersela, risentiva le trepide gioie del plasmarne e vivificarne i concetti ${ }^{29}$.

Le suggestioni del palcoscenico hanno dunque un'inevitabile e interessante ricaduta anche in ambito critico e letterario, dove si riconosce da più parti che questo Dante di cartapesta fatto rivivere sotto i lumi della ribalta è in grado

27. Si rimanda a Petrini (2012: 310).

28. Grandi (a cura di) (I955: 249).

29. [Sia], Appendice. Ciarle del lunedi, in "Il Risorgimento", 23 aprile I856 (cfr. Petrini 20I2: I84). 
di comunicare con finezza e profondità la poesia della Commedia. Arrigo Bocchi, giornalista e poeta, scrive ad esempio nel I839:

questo attore mette riverenza ai più culti ingegni, i quali possono ammirare piuttosto che contendergli la vittoria nello intendere, ed esporre in pari tempo, i pensieri sublimissimi di quell'immortale italiano ${ }^{30}$.

Mauro Macchi, nel 1856, parla di Modena come di un "uomo [...] che per la vastità dell'ingegno, e per gli studj che fece su le cantiche del fiero ghibellino, è universalmente acclamato come il migliore commento del gran poeta" ${ }^{\text {II }}$. Un testimone di spicco, quale Collodi, concorda che: "Fra i mille e mille commenti che esistono della Divina Commedia il più vero di tutti, il più efficace di tutti, è incontestabilmente Gustavo Modena" (Collodi I892: I03). Anche l'accademia più ufficiale finirà per riconoscere una tale eccellenza, come conferma, molti anni dopo la morte dell'interprete, per esempio Vincenzo Andrei: "Nelle aule magne, massime nella Atene d'Italia, la dotta parola del prof. Guido Mazzoni lo designava come un Dantofilo, e quale manifestatore scenico che aveva rimontato alle sorgenti del vero"32. La partita fra filologia e divulgazione - inaugurata negli anni ' 30 dalle polemiche di Mazzini contro i critici-imbalsamatori da neutralizzare ricorrendo a nuovi linguaggi e a nuovi media per tenere in vita questo padre della patria - si chiude in parità: un grande dantista legittima Modena in quanto "manifestatore scenico" della verità della Commedia. Eppure la sua eterodossa ermeneutica resta alla fine un miracolo e un paradosso imperscrutabile:

Rinunciamo [...] a descrivere con che tono d'ispirazione, con che profondo e semplice accento, con che musica ineffabile egli dicesse il verso e la terzina di Dante, innestando un ritmo pieno di maestà e di unzione alla diversa espressione che richiedevano i fremiti di Ugolino, i sospiri di Francesca, le imprecazioni alla simonia dei papi, le astruse trasformazioni dei serpenti nei canti dell'Inferno, contentandoci solo di asserire che non mai più potente invito fu fatto a studiare quel sommo, non mai miglior commento a intenderlo; e se le sue note si fossero potute scrivere, sarebbero rimaste perennemente affisse a quei canti, come ai Treni agl'Improperii le note del Palestrina. Quindi la recitazione di Dante è un segreto che Gustavo Modena portò nella tomba Ardito (I868: I2).

In verità Modena perde la sua battaglia politica e teatrale e, come si è detto, muore, povero e deluso, alla vigilia di un' unificazione realizzatasi infine per via monarchica, e solo grazie a numerosi compromessi gravidi di pericolose conseguenze. Il progetto di "seppellirne le sante ossa stanche appiè di Dante" in Santa Croce, trasferendole dall'oscuro tumulo che gli era toccato nel cimi-

30. A. Bocchi, Declamazione, in "Il Vaglio", 21 settembre 1839 (cfr. Petrini 20I2: 190).

31. Il giudizio è espresso in una lettera del 1856 indirizzata al direttore della rivista "La Ragione" (cfr. Petrini 20I2: 190).

32. Andrei 1902 (in Petrini 2012: 190). 
tero acattolico di Torino, resta senza esito ${ }^{33}$ nell'Italia postunitaria, che presto lo dimentica. La storia teatrale e politica della penisola sarà lontanissima da quella che egli aveva vagheggiato; e nondimeno il format teatrale di sua invenzione - rivisto e corretto nei contenuti e nei fini - conoscerà una straordinaria fortuna, parallela all'allarmante quantità di drammi e libretti che ancora a fine secolo si continuano a ricavare dalla Commedia ${ }^{34}$.

La svolta radicale che inaugura il culto nazionalistico dantesco, prima monarchico e poi fascista ${ }^{35}$, si identifica con le celebrazioni del centenario del I865, organizzate a Firenze, nuova capitale del regno, e culminate nello scoprimento di quel monumento al poeta "che si preparava in Firenze" già in vista dell'anniversario del $182 \mathrm{I}^{36}$, e che, più di quarant'anni dopo, realizza finalmente lo scultore ravennate Enrico Pazzi. Quell'evento pubblico, saturo di teatro e di teatralità, ebbe proporzioni e risonanza straordinarie per l'epoca, rinnovando le forme tipiche della festa rinascimentale italiana (e fiorentina in particolare) in chiave di propaganda monarchica e patriottica, e ridisegnò il profilo del poeta per gli anni a venire, facendone un prototipo di italianità e di cosmopolitismo pronto per nuovi utilizzi. La città lorenese era stata rimessa a nuovo sul piano urbanistico come una moderna capitale europea, ma si trattava ancora di un contenitore pressoché vuoto, e il poeta - le cui ossa la città di Ravenna si era rifiutata come sempre di restituire all'ingrata Firenze ${ }^{37}$ - offriva un formidabile riempitivo da sfruttare. Pur in mancanza di adeguate risorse finanziarie, si mise in moto una macchina organizzativa accuratissima, costellata di eventi, di pubblicazioni d'occasione e di spettacoli, di cui l'Alighieri fu il brand di straordinario impatto: "Il I4 maggio sarà memorabile nella storia degli italiani: e resterà ricordo glorioso della gioia di un giorno che cancella un dolore di secoli. Spettacolo maestoso: che compendia tutte le nostre istorie nell'assemblamento lieto di tante genti fin oggi divise, e vaticinava alla madre patria serenità e sicurezza di futuri destini" ${ }^{8}$.

33. Cfr. Grandi (a cura di) (1955b: 214).

34. Per una prima ricognizione del fenomeno si fa riferimento alla voce "Teatro" curata da G. Antonucci nel vol. V dell' Enciclopedia dantesca, (Antonucci 1984).

35. Sulla strategia dantesca mussoliniana, che prevedeva, fra l'altro, la costruzione di un "Danteum" (poi non realizzato) ai Fori Imperiali, si veda Chiodi (20I2).

36. Gli eventi politici impedirono di realizzare, in quella circostanza, il progetto a cui il giovane Leopardi aveva dedicato le strofe appassionate della canzone Sopra il monumento di Dante che si preparava in Firenze, composta fra il settembre e l'ottobre i818, quando dalla città era partita, allo scopo, una pubblica sottoscrizione nazionale. Sulle vicende ottocentesche "dell'allegoria della donna-patria" dantesca e petrarchesca, cfr. Serra (2012).

37. Come è ben noto, le spoglie del poeta - sempre richieste invano dalla madrepatria - sono custodite a Ravenna (non senza polemiche e peripezie plurisecolari), per cui a Firenze ci si era infine rassegnati, nel I829, a costruire in Santa Croce un cenotafio neoclassico, sormontato da una sua statua pensosa e severa, con la Poesia in lacrime china sul sarcofago.

38. "La Nazione", is maggio I864. Questo trionfale auspicio, espresso l'anno precedente sulle pagine del quotidiano fiorentino, sarà il filo conduttore del complesso "lancio" della 
La manifestazione monopolizza per tre giorni, dal i4 al i6 maggio, la vita di una Firenze trasformata da apparati effimeri in un gigantesco palcoscenico gremito di ghirlande e bandiere.

Il primo giorno un corteo composto da rappresentanti di province, comuni, istituzioni culturali, ma anche "giornalisti e attori" ${ }^{39}$, sfila per due ore e mezzo da piazza Santo Spirito a piazza Santa Croce; fra i gonfaloni spiccano le insegne di Roma, Venezia, Firenze e Ravenna. Alle in arriva anche Vittorio Emanuele, prendendo posto su un palco d'onore accanto a numerose delegazioni straniere. La statua, collocata al centro della piazza, raffigura il "fiero ghibellino" atteggiato a "generoso sdegno" (quella che incombe, adesso, è la questione romana) e troneggia su un basamento quadrangolare riccamente scolpito con vari bassorilievi alternati agli stemmi delle città italiane. Intorno le è stato costruito un anfiteatro di legno in grado di ospitare i80oo spettatori, decorato con trentotto bassorilievi dipinti che hanno per tema la vita del poeta (non casualmente il personaggio dell'autore comincia a sovrastare i contenuti dell'opera), e anche quaranta dei suoi biografi, traduttori e commentatori (da Jacopo della Lana e Pietro Alighieri, fino a Foscolo e a Colomb de Batines) si meritano altrettanti ritratti.

La città è disseminata di iscrizioni che costruiscono un itinerario ideale delle passate glorie fiorentine e dei personaggi illustri che vi hanno transitato e soggiornato: non solo segnalando i luoghi danteschi, come il perimetro delle case degli Alighieri, o la casa natale di Beatrice Portinari, ma anche quelli toccati da altri aspiranti candidati da accogliere nel costituendo parterre di glorie "italiane", come ad esempio Carlo Goldoni "scrittore arguto e vivace nell'interpretare la Natura, salutato principe della commedia italiana"40. Passato e presente si fondono nella Nazione riunificata, e l'arco d'ingresso a piazza Santa Croce è sormontato dalla solenne iscrizione "Italiani / Onorate l'altissimo poeta / L'omaggio che rendete a lui / Vendica l'oblio di sei secoli / E attesta al mondo / Che siete Nazione"4I. Il fitto programma di questa modernissima "tre giorni" comprende appuntamenti destinati a tutti gli strati sociali e disseminati un po' in tutta la città: concerti e cori (che eseguono inni e musiche in gran parte composti per l'occasione), cavalcate in costume; regate in Arno, cuccagne a premi e corse di cavalli alle Cascine; in via Vittorio Ema-

capitale nel segno dantesco, a cui fanno eco analoghe manifestazioni in altri centri della penisola: lo scoprimento della statua di Dante scolpita dallo Zanoni nella piazza dei Signori a Verona, il battesimo ufficiale dell'ex "ponte dell'Impossibile" come "ponte Dante" a Treviso, lo scoprimento di busti, medaglioni e lapidi in numerosi centri grandi e piccoli, soprattutto del Veneto.

39. Fra le numerose pubblicazioni e rassegne stampe dedicate agli eventi scegliamo di riferirci qui alla Guida ufficiale per le feste del centenario di Dante Alighieri nei giorni I4, Is e I6 maggio I865, (Guida ufficiale... I865).

40. Guida ufficiale... (I865: 20).

4I. Guida ufficiale... (1865: 28). 
nuele un gran torneo ricorda la pace del $\mathrm{I}_{3} \mathrm{O} 4$ fra guelfi e ghibellini, preceduto dall'esibizione di un funambolo in costume di araldo che fa "l'uomo volante, spargendo fiori e rami d'olivo in segno di pace e di allegrezza"; una tombola pubblica si svolge in piazza Indipendenza a beneficio degli asili di carità; balli popolari si tengono agli Uffizi, mentre le élites danzano al ricevimento ufficiale del casino Borghese di via Ghibellina. Le principali istituzioni culturali cittadine - come l'Università, la Crusca o l'“Ateneo Italiano" - offrono il loro contributo in forma di accademie musicali, pubbliche letture, prolusioni (fra cui una di Carducci), esposizioni di ogni tipo...

La sera di martedì 16 si tiene una "accademia di quadri viventi con declamazioni scelte della Divina Commedia nel Regio Teatro Pagliano" di via Ghibellina (che più tardi sarà intitolato a Verdi). L'evento eccezionale riunisce i tre grandi della scena teatrale italiana: Adelaide Ristori arriva da Parigi, Ernesto Rossi da Milano e Tommaso Salvini da Napoli ${ }^{42}$. Memori del loro maestro, ma fedeli ai tempi nuovi e al proprio monumentale prestigio artistico, i tre si producono in eccellenti letture drammatizzate del poema (accompagnate da mimi e da musiche composte per la circostanza dal maestro Bettazzi) remote dalla feroce ruvidezza delle dantate modeniane sia sul piano dello stile che dei contenuti. La scaletta della serata prevede tredici quadri: si comincia dal saluto di Beatrice nella Vita Nova (in musica); quindi si avvicendano cinque pezzi sull'Inferno, con Salvini che "declama" i brani sulla lupa e sul Conte Ugolino, e Rossi su Farinata e sui ladri, mentre la Ristori recita, ovviamente, la storia di Francesca. Nella seconda parte i tre pezzi sul Purgatorio avvicendano la Pia della Ristori, il Sordello di Rossi e la porta del Purgatorio di Salvini; negli ultimi quattro relativi al Paradiso la Ristori recita Piccarda, mentre Gaetano Gattinelli declama i versi di Cacciaguida e di San Pietro; un coro chiude in musica con l'apoteosi dell'autore. La chiave dello spettacolo è interamente politica, come testimonia tra l'altro Salvini con una certa malizia:

Il teatro presentava un aspetto imponente! Il re Vittorio Emanuele, il Senato, gli ambasciatori, i deputati, l'aristocrazia, l'armata, il foro, le arti, l'industria, il commercio, tutte le caste della società erano rappresentate; e quel vastissimo locale fu incapace di contenere l'immensa folla che si addensava inutilmente alle porte del teatro per poter godere lo spettacolo. Come illustratore del primo canto fui naturalmente il primo a presentarmi sulla scena. Un applauso di simpatia accolse la mia entrata. Quando venne il punto nel quale il Divino Poeta simboleggia nella Lupa la Curia

42. Nominato da poco presidente della neo-nata Società di mutuo soccorso fra gli artisti drammatici creata a Napoli, egli rappresenta idealmente tutti gli attori italiani. E' abbastanza significativo sottolineare che gli artisti chiamati ad animare questa grandiosa manifestazione non ricevettero neanche un rimborso spese, come sottolinea un po' piccato Ernesto Rossi nelle sue memorie (Rossi 1887), ricordando di aver regalato al suo servitore il modesto orologio ("non un nome, non una cifra, non una data sopra!") recapitatogli qualche mese dopo a titolo di compenso. 
romana e prende a dire: "Molti son gli animali a cui s'ammoglia, / e più saranno ancora, infin che 'l veltro / verrà, che la farà morir con doglia!", guardai fissamente il Re e restai qualche secondo senza parlare. L'uditorio comprese simultaneamente l'allusione e un uragano d'applausi eruppe che non aveva più fine ${ }^{43}$.

Il re è, per così dire, il referente naturale della festa; già il giorno prima l'oratore ufficiale, il professor Augusto Conti, che aveva inaugurato le manifestazioni al palazzo del Podestà, lo aveva accolto a Firenze, consegnandogli, in nome dello stesso poeta, una spada d'onore con incisa la terzina "vieni a veder la tua Roma che piange"44. Lo spettacolo del teatro Pagliano lo chiama ancora direttamente e un po' brutalmente in causa: il Veltro tanto atteso è diventato lui, e il poeta ribelle ha cambiato casacca, subendo una mutazione che sarà irreversibile.

Nei ricordi di Salvini il sovrano appare disorientato (e, secondo la sua impressione "avrebbe desiderato di trovarsi a caccia, anziché a teatro"), ma fa naturalmente buon viso a cattivo gioco calandosi in panni certamente poco familiari: "restò esitante parecchio tempo, finché gli fu forza di alzarsi e ringraziare più volte il pubblico". Il maquillage dell'Italia unificata era appena agli inizi, ma tutti gli elementi erano stati posti sul tavolo. Inoltre queste solenni celebrazioni pubbliche ebbero un'appendice importante, qualche giorno dopo, con la recita della Francesca da Rimini di Pellico al teatro Niccolini, che riunì eccezionalmente le tre grandi star presenti in città. L'evento fu il risultato di abili mediazioni impresariali e delle forti pressioni di un comitato pubblico formatosi per la circostanza, che riuscirono a neutralizzare le tradizionali rivalità reciproche fra i tre divi, interessati peraltro a qualche utile, vista l'avarizia delle autorità ufficiali. Fu un record di incassi (I5000 lire per due serate) ${ }^{45}$. I giochi erano fatti. Il divino poeta era pronto per le aule universitarie, le targhe di marmo e i monumenti pubblici, ormai grandioso e sterilizzato come, di lì a poco, tanti altri "grandi", con in testa Garibaldi. La sua popolarità, costruita da Gustavo Modena per forgiare la coscienza politica delle masse incolte, era pronta per trasferirsi nel cinema: gli albori della cinematografia italiana vedono replicata in celluloide l'antologia della Commedia canonizzata in teatro ${ }^{46}$ e il primo lungometraggio italiano prodotto dalla Milano Film,

43. Salvini (1895) (in Giammusso 1994: II2)

44. Le feste... (1865).

45. Le aspettative erano altissime circa, soprattutto, l'assegnazione dei ruoli, che poteva porre qualche problema: Salvini accettò invece di interpretare il "cattivo" Lanciotto senza apparenti resistenze, ma ne fece un marito ferito e innamorato, che sovrastò Paolo, rubando in parte la scena a Rossi.

46. Ricordiamo, fra gli altri, nel 1908 Il conte Ugolino di Giuseppe de Liguoro, la Pia de' Tolomei di Mario Casarini e la Francesca da Rimini di Mario Marais; nel 1909 il Conte Ugolino di Giovanni Pastrone; nel rgro un'altra Pia de’ Tolomei di Gerolamo Lo Savio, e nel ı9ı Il Paradiso (visioni dantesche) di Giovanni Pettine. 
con un ingente investimento economico e un cast di 150 comparse, è dedicato per l'appunto all'Inferno. Francesco Bertolini, Giuseppe De Liguoro e Adolfo Padovan realizzano, nel I9II, un kolossal con 54 quadri animati, ispirati alle celeberrime illustrazioni della Commedia di Gustave Doré, dove una serie di effetti speciali restituiscono vividamente sullo schermo le illusioni che Modena costruiva con la forza della parola, del corpo e della mimica: sovrimpressioni per la bufera che travolge i lussuriosi, montaggi per i ladri mutati in serpenti, esposizioni multiple per la testa recisa di Bertrand de Born.... Lo spettacolo continua.

\section{BiBLIOGRAFIA}

Andrei, V., I899, Gli attori italiani da Gustavo Modena a Ermete Novelli Studio critico, Firenze, Elzeviriana.

-, 1902, Dante divinatore. Ampliamento di una lettura dantesca tenuta nel reale Collegio Cicognini in Prato e nel reale Istituto Nazionale in Firenze, Firenze.

Antonucci, G., I984, "Teatro" in Enciclopedia dantesca V, Roma, Istituto della Enciclopedia Italiana, pp. 530-533

Ardito, P., I868, Gustavo Modena e l'arte sua per Luigi Bonazzi: saggio critico, Sanseverino-Marche, Società Editrice C. Corradetti.

Barocchi, P., (et al.), 1974, Romanticismo storico Celebrazioni per il Centenario di Francesco Domenico Guerrazzi), Firenze, Centro Di/edizioni, pp. 4I- 55 .

Battistini, A., 20oI, "Il modello e le suggestioni letterarie: Dante nella tradizione della letteratura e nella cultura popolare", in "Per coglier miglior acque...". Bilanci e prospettive degli studi danteschi alle soglie del nuovo millennio, Roma, Salerno, pp. 443-448;

—, 2008, "Miti, leggende e personaggi di Romagna nei primi commentatori della Commedia", in Dante e la fabbrica della "Commedia", Ravenna, Longo,2008, pp. 283-303.

Chiodi, S., 20I2, "Dante fascista e i suoi disegni", in S. Luzzatto e G. Pedullà (a cura di), Atlante della Letteratura Italiana. III. Dal Romanticismo a oggi, Einaudi, Torino, pp. 67I-675.

Collodi, (C. Lorenzini), I892, Divagazioni critico-umoristiche, (a cura di G. Rigutini), Firenze.

Costa, G., 2002, "Il risveglio dell'attenzione alla cultura italiana", in Storia della Letteratura Italiana, XII. La Letteratura Italiana fuori d'Italia, Roma, Salerno, pp. 547-550.

Dionisotti, C., I97I, "Varia fortuna di Dante", in id. Geografia e storia della letteratura italiana, Torino, Einaudi, pp. 255-303.

Le Feste..., I865, "Le feste per la celebrazione del VI centenario di Dante Alighieri", in L'Opinione, Torino-Firenze-Roma [I5 maggio I865].

Giammusso, M.,1994, Il Dante di Gassman. Cronaca e storia di un'interpretazione della "Divina Commedia", Milano, Mondadori.

Grandi, T., (a cura di), I955a, Epistolario di Gustavo Modena, Roma.

-, (a cura di), 1955b, Scritti e discorsi di Gustavo Modena, Roma, Vittoriano. 
-, I968, Gustavo Modena attore e patriota, Pisa, Nistri Lischi.

Guida ufficiale..., I865, Guida ufficiale per le feste del centenario di Dante Alighieri nei giorni I4, Is e I6 maggio I865, Firenze, M. Cellini.

Mango, L., 20I2, "I dialoghi politici di Gustavo Modena”, in Petrini (a cura di) (2012) p. 85-99.

Mazzini, G., s.d., "Dell'amor patrio in Dante", in Scritti scelti, (a cura di J. White Mario), Firenze, pp. 6-I5.

Meldolesi, C., I97I, Profilo di Gustavo Modena. Teatro e rivoluzione democratica, Roma, Bulzoni.

Petrini, A., 20I2, Gustavo Modena. Teatro, arte, politica, Pisa, ETS.

-, (a cura di), 2012, Ripensare Gustavo Modena. Attore e capocomico, riformatore del teatro, fra arte e politica, Roma, Bonanno editore.

Pieri, M., I995, "Dalla Commedia dell'Arte al Sillabo: varie sorti di Torquato mattatore", Italianistica, XXIV / 2-3, pp. 593-6I4.

-, 20Io, "Come si inventano le tradizioni: il Medioevo antagonista di Mistero buffo", Revue des études italiennes, 56 / 3-4, pp. I85-197.

Pietrini, S., 20I2, "La recitazione grottesca e l'eredità mancata di Gustavo Modena", in A. Petrini (a cura di), 2012, pp. 195-212.

Puppa, P., 20I2, "Gustavo Modena dramaturg e lettore di Dante", in Petrini (a cura di), 2012, pp.. I79-I94.

Querci, E. (a cura di), 2oII, Dante vittorioso. Il mito di Dante nell'Ottocento, TorinoLondra-Venezia-New York, Allemandi.

Randi, E., 20I2, "Il Luigi XI di Gustavo Modena", in Petrini (a cura di), pp. I65-I78.

Rasi, L., I905, I comici italiani, biografia, bibliografia, iconografia, II, Firenze, Bocca.

Rossi, E., I887, Quarant'anni di vita artistica, Firenze, Tipografia Editrice Niccolai.

Salvini, T., I895, Ricordi e impressioni, Milano, Fratelli Demolard Editori.

Sanguanini, B., 1989, Il pubblico all'italiana. Formazione del pubblico e politiche culturali tra Stato e Teatro, Milano, Franco Angeli.

Serra, F., 20I2, "Povera Italia", in S. Luzzatto e G. Pedullà (a cura di), Atlante della Letteratura Italiana, vol. III Dal Romanticismo ad oggi, a cura di T. Scarpa, Torino, Einaudi, 20I2, pp. 8-13. 